\title{
Nanoscale surface roughening in ultrathin aluminum films
}

\author{
D. Aurongzeb, E. Washington, M. Basavaraj, J. M. Berg, H. Temkin, and M. Holtz ${ }^{\text {a) }}$ \\ Nano Tech Center, Texas Tech University, Lubbock, Texas 79409
}

(Received 6 April 2006; accepted 24 August 2006; published online 13 December 2006)

We report studies of the formation of aluminum structures, with nanometer length scales, in ultrathin layers deposited on glass and analyzed using atomic force microscopy. Surface roughness, lateral cutoff length, and surface scaling exponent are all found to vary systematically with Al thickness. The initial nanocrystal formation is described by stress-energy minimization. We interpret the observed scaling behavior based on the kinetic theory of roughening. For very thin layers $(<50 \mathrm{~nm})$ the roughness is consistent with a $\nabla^{4} h$ growth factor, where $h$ is surface height. For thick layers $(>50 \mathrm{~nm})$ the $\nabla^{2}(\nabla h)^{2}$ conserved mechanism is dominant. Substrate stress is not found to influence the scaling exponent through the kinetic theory. (C) 2006 American Institute of Physics. [DOI: $10.1063 / 1.2365388$ ]

\section{INTRODUCTION}

Metallic thin films have broad technological relevance with applications in memory, microelectronics, and photonics. Deposition of thin metal films by physical vapor deposition results in the formation of self-assembled nanostructures. These structures can form upon postdeposition annealing, ${ }^{1}$ in situ at elevated substrate temperature, ${ }^{2}$ or at room temperature. ${ }^{3,4}$ Depending on interaction forces internal to the thin-film and with the substrate, and surface diffusion across substrate and forming facets, the structures may manifest as islands or by the subtle formation of roughened surfaces. Chemical interactions between the layer and substrate may also be important, as in the case of silicides.

A wide variety of surfaces and interfaces exhibit roughness associated with scaling. However, there remains an unanswered question whether similar surface morphologies, obtained under different physical conditions, possess a common basis. Roughness can be generated by factors such as noise, geometrical shadowing, the Ehrlich-Schwoebel barrier, and stress originating at the layer/substrate interface. Recent studies on the formation of nanodots on substrates have generated interest in the physics of self-assembly and provide an interesting venue for studying surface roughening. ${ }^{5,6}$ Atomic force microscopy (AFM) allows us to study surfaces with $\sim 0.1 \mathrm{~nm}$ height and $\sim 10 \mathrm{~nm}$ lateral resolution. Important aspects of the physical processes important to nanodot formation include the surface energy of the substrate material, the surface and bulk energies of the structure itself, and the interaction between the substrate and forming material. ${ }^{7-10}$ In numerous situations, heterogeneous interfaces may result in stress due to mismatches at the interface, an effect known to influence the deposited layer morphology. ${ }^{11}$

Surfaces are characterized by root-mean-square (rms) roughness or interface width $w$ defined as

$$
w^{2}=\left\langle[h(\mathbf{r})-\langle h(\mathbf{r})\rangle]^{2}\right\rangle,
$$

where $h(\mathbf{r})$ is surface height at position $\mathbf{r}=(x, y)$ and $\langle\cdots\rangle$ denotes spatial average. Layers formed under nonequilibrium

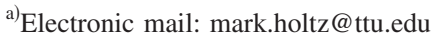

conditions are expected to develop self-affine surfaces, with interface widths which depend on time $t$ and length scale $L$ sampled according to the Family-Vicsek function, ${ }^{12}$

$$
w(L, t)=L^{\alpha} \varphi\left(t / L^{\alpha / \beta}\right),
$$

where $w(L) \propto L^{\alpha}$ for $t / L^{\alpha / \beta} \rightarrow \infty$ and $w \propto t^{\beta}$ for $t / L^{\alpha / \beta} \rightarrow 0$. Parameter $\alpha$ is the roughness exponent and $\beta$ is the growth exponent. Self-affine surfaces are also characterized by an upper limit to scaling or cutoff length $\xi$ above which $w$ no longer scales as $L^{\alpha}$. The correlation length also increases with time according to $t^{\beta / \alpha}$, where the ratio $\alpha / \beta$ is referred to as the dynamic scaling exponent.

Theoretical treatments of nonequilibrium film growth rely on kinetic roughening to describe the scaling behavior. The evolution of the growing surface $h(\mathbf{r}, t)$ is described by

$$
\frac{\partial h}{\partial t}=F[h]+\Phi+\eta,
$$

where the functional $F[h]$ is composed of terms which obey scaling relations. These include the Mullins term $\nabla^{4} h$, which stems from the curvature dependence of the chemical potential, the conserved Kardar-Parisi-Zhang (CKPZ) term $\nabla^{2}(\nabla h)^{2}$, which originates from the slope dependent surface growth, and a $\nabla^{2} h$ factor, which describes the presence of Ehrlich-Schwoebel barriers at crystal facet edges. $\Phi$ is the deposition flux and $\eta(\mathbf{r}, t)$ accounts for random (noise) processes related to deposition.

In this paper, we study ultrathin aluminum layers deposited at room temperature to examine the noncrystallographic influence of an amorphous substrate on the formation of nanometer-scale features. While substantially more is known about stress developed in crystalline layers produced on crystalline substrates, particularly when grown at elevated temperatures, few papers report deposition of amorphous layers on crystalline substrates ${ }^{13}$ or crystals formed on an amorphous substrate. ${ }^{14}$ In the ultrathin $\mathrm{Al}$ layer regime, where interface stress plays an important role in the film morphology, we observe a roughness exponent $\alpha>1$. This is predicted by kinetic theory, ${ }^{15}$ although there are few observations of roughness exponents near to and greater than unity. ${ }^{3,16,17}$ 


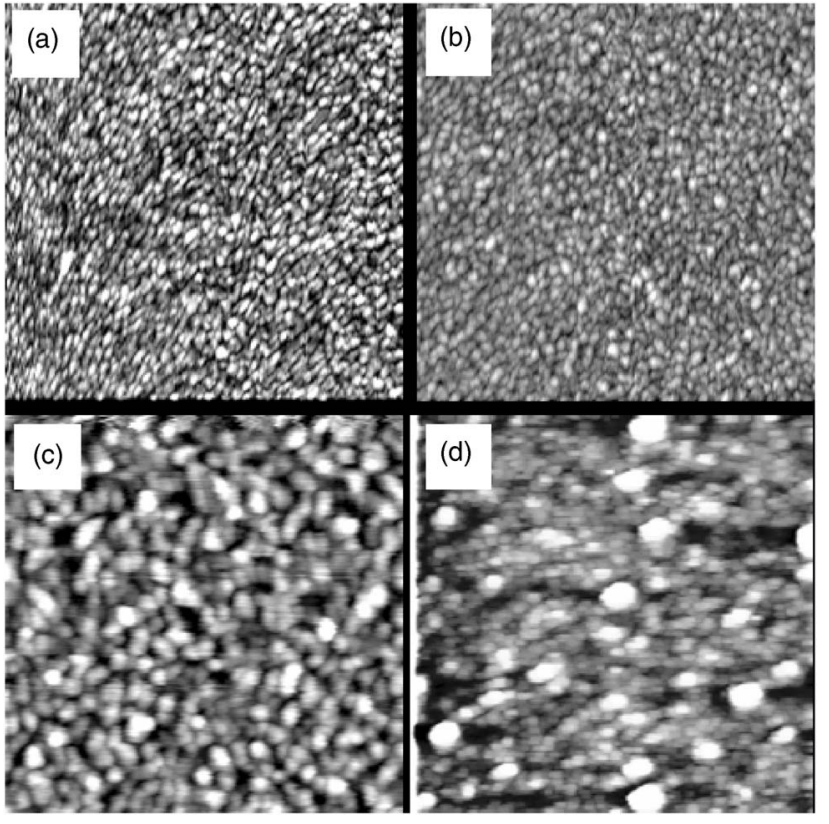

FIG. 1. AFM images of Al layers with average thickness/full vertical gray scale: (a) $6 \mathrm{~nm} / 4.0 \mathrm{~nm}$, (b) $10 \mathrm{~nm} / 9.0 \mathrm{~nm}$, (c) $17 \mathrm{~nm} / 9.5 \mathrm{~nm}$, and (d) $40 \mathrm{~nm} / 9.5 \mathrm{~nm}$. The images are $1 \times 1 \mu \mathrm{m}^{2}$.

\section{EXPERIMENTAL DETAILS}

Aluminum layers were deposited on commercial glass wafers $(w \sim 1 \mathrm{~nm})$ using electron beam evaporation under high vacuum (base pressure of $\sim 10^{-8}$ torr). Deposition pressure ranges from $10^{-7}$ to $10^{-6}$ torr. Thickness was varied from 2 to $350 \mathrm{~nm}$. Substrate temperature remained below $100{ }^{\circ} \mathrm{C}$, and no postdeposition anneal was carried out.

Surface properties are investigated using AFM. We define the layer-substrate interface as $h=0$ and average height $\bar{h}$ is the nominal layer thickness. The surface-roughness spectrum or power spectral density $(S)$ used to examine scaling. ${ }^{6}$

$$
S(\mathbf{k})=\frac{1}{L^{2}}\left|\int(h(\mathbf{r})-\bar{h}) e^{2 \pi i \mathbf{k} \cdot \mathbf{r}} d \mathbf{r}\right|^{2},
$$

where the spatial frequency $\mathbf{k}$ is $k \hat{\mathbf{r}}$ for surfaces which are isotropic in $(x, y)$, as presented here. The valid spatial frequency range is determined by the AFM scan length ( $L_{\text {scan }}$ $=1 \mu \mathrm{m})$ and AFM tip radius $\left(R_{\text {tip }}<10 \mathrm{~nm}\right)$, such that $5 / L_{\text {scan }} \leqslant k \leqslant 1 / R_{\text {tip }}$. Commercial tips (conical) are used with two different AFM instruments. The tip dimensions are $\sim 10 \mathrm{~nm}$ estimated using scanning electron microscope images. Checks of tip sharpness are performed routinely, and tips are rejected when evidence of wear is observed. The images shown in Fig. 1 were taken in contact mode with $256 \times 256$ pixel resolution. The pixel dimension of $\sim 4 \mathrm{~nm}$ is smaller than the tip radius, so that the latter is relevant in determining the valid range of $k$ in Eq. (4). Function $S(k)$ can then be used to extract scaling behavior according to $S(k)$ $\propto k^{-\varepsilon}$, where $\varepsilon$ is related to the height correlation function exponent through $\alpha=(\varepsilon-d) / 2$ with $d$ the dimensionality.

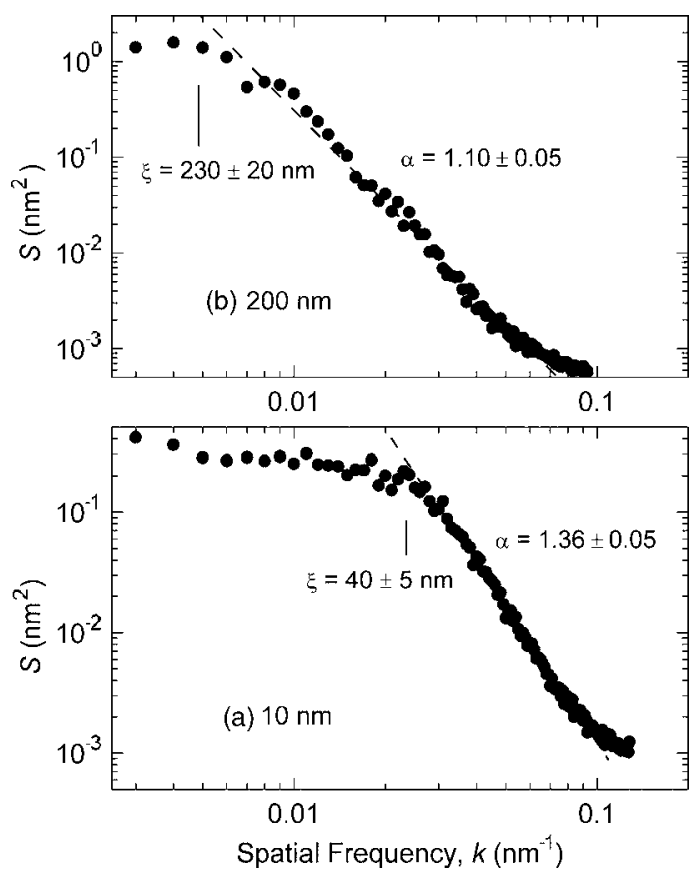

FIG. 2. Example power spectra for surface measurements in Fig. 1.

\section{RESULTS}

Figure 1 shows images of $\mathrm{Al}$ films with varying thickness obtained using AFM. Very thin layers exhibit a finegrained structure with small lateral size. The grain size grows with layer thickness up to $40 \mathrm{~nm}$. Gradient analysis ${ }^{18}$ shows the surface normal for these thin layers to have a broad distribution, indicating locally sloped surfaces. In-plane gradient analysis shows no angular correlation of the nanocrystals expressing the lack of faceting or in-plane alignment. The $\bar{h}>100 \mathrm{~nm}$ layers exhibit larger polycrystals, as seen in Fig. 1(b), which are highly oriented. Between 40 and $100 \mathrm{~nm}$ we observe the fine-grained morphology mixed with larger, but poorly formed, polycrystals as shown in Fig. 1(d).

In Fig. 2 we show $S(k)$ for several layers. Below the cutoff frequency $k_{c}$ in each panel we observe a weak dependence on $k$, consistent with a lack of scaling over the length regime $L>\xi=1 / k_{c}$. The cutoff length $\xi$ is generally associated with the average feature size seen in the image. For spatial frequencies above $k_{c}, S(k)$ exhibits the rapid drop characteristic of scaling. Least-squares linear fits to the two ranges allow us to determine $k_{c}$ and $\varepsilon$, and subsequently the exponent $\alpha$ in the high-frequency scaling range.

In Fig. 3 we summarize the dependence of $w, \xi$, and $\alpha$ on deposited $\mathrm{Al}$ thickness. The roughness and cutoff length values rise rapidly up to $\bar{h} \sim 100 \mathrm{~nm}$, above which they saturate at 6 and $200 \mathrm{~nm}$, respectively, describing statistically smooth surfaces. Below $\bar{h} \sim 5 \mathrm{~nm}$ the layers are composed of discontinuous island structures, and our measurements constitute a superposition of substrate $(\alpha \sim 0)$ and nanodot characteristics. Above $5 \mathrm{~nm}$ the results are divided into two types of behavior. The thinnest films studied show small features, growing roughness, and changing scaling exponent. The scaling exponent rises rapidly for ultrathin layers and reaches $\alpha=1.42 \pm 0.15$ for $\bar{h}$ between 5 and $20 \mathrm{~nm}$, before returning 


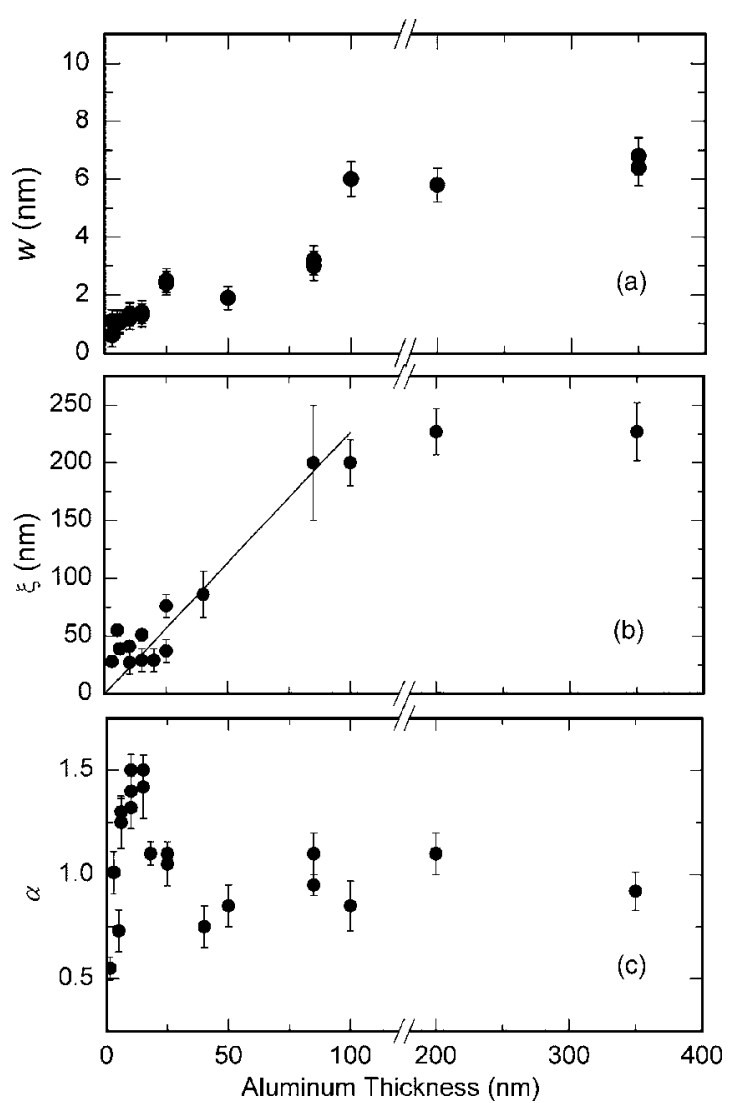

FIG. 3. (a) Surface roughness, (b) lateral cutoff length, and (c) scaling exponent vs aluminum layer thickness.

to $\alpha \sim 1$ above $50 \mathrm{~nm}$. For $\bar{h}<100 \mathrm{~nm}$ we obtain $\beta$ $=0.46 \pm 0.05$. For $\bar{h} \geqslant 100 \mathrm{~nm}$ we find $\beta=0.27 \pm 0.10$. Above $\bar{h} \sim 100 \mathrm{~nm}$ the films exhibit a steady dependence in the quantities $w, \xi$, and $\alpha$.

\section{DISCUSSION}

Observation of a scaling exponent $\alpha>1$ has been reported in only a few cases. ${ }^{3,16,17}$ One possible factor influencing the scaling exponent is the interface interaction between the substrate and the Al layer. Good adhesion of the $\mathrm{Al}$ to the glass originates from the $\mathrm{Si}-\mathrm{O}-\mathrm{Al}$ initial bonding, and interface strain will induce stress on the Al. The interaction between is known to induce the formation of nanocrystals through mismatches in the surface energy densities. According to Refs. 7 and 19, the optimal lateral size

$$
x_{0}=e \varphi z \exp (\Gamma / c z)
$$

is obtained upon minimizing the nanodot energy taking into account its bulk stress energy $\left(\sigma_{b}\right)$, surface energy of the top and sidewalls ( $\gamma_{t}$ and $\gamma_{s}$, respectively), the substrate surface $\left(\gamma_{\text {sub }}\right)$, and the island-substrate interface energy $\left(\gamma_{i}\right)$. Island height is denoted as $z$ and the other terms in Eq. (5) are $\varphi$ $=e^{-3 / 2} \cot \theta, \quad \Gamma=\gamma_{s} \csc \theta-\left(\gamma_{t}+\gamma_{i}+\gamma_{\mathrm{sub}}\right) \cot \theta$, and $c=\sigma_{b}^{2}(1$ $-\nu) / 2 \pi \mu$. Here, $\theta$ is the contact angle between the island sidewall and the substrate, $\nu$ is the Poisson ratio of $\mathrm{Al}$, and $\mu$ is the shear modulus (Table I). For very thin Al deposition, the contact angle is $\sim 15^{\circ}$ from the AFM measurements. In Fig. 3(b) we show this calculation to be in good agreement
TABLE I. Relevant physical quantities of nickel and glass.

\begin{tabular}{ccc}
\hline \hline Quantity & Value & Reference \\
\hline$\gamma_{\mathrm{s}}, \gamma_{t}$ & $0.81-1.05 \mathrm{~J} / \mathrm{m}^{2}$ & 24 \\
$\gamma_{i}$ & $-1.28 \mathrm{~J} / \mathrm{m}^{2}$ & 24 \\
$\gamma_{\text {sub }}$ & $1.6 \mathrm{~J} / \mathrm{m}^{2}$ & 25 \\
$D_{s}$ & $2.5 \times 10^{-6} \mathrm{~m}^{2} / \mathrm{s}$ & 26 \\
$\mu$ & $26 \mathrm{GPa}$ & 27 \\
$M$ & $76 \mathrm{GPa}$ & 27 \\
$\nu$ & 0.16 & 27 \\
Lattice constant & $4.03 \AA$ & This work \\
\hline \hline
\end{tabular}

with the cutoff length results up to thickness of $100 \mathrm{~nm}$ and using $z=\bar{h}$ in Eq. (5), which is reasonable for very thin layers. The value used for $\sigma_{b}$ is calculated using a $0.3 \%$ mismatch strain of the Al, obtained from (111) and (200) x-ray diffractions, and the elastic modulus. Strain arises from the interface formed with the substrate, and is subsequently present in the nanodots. The calculated dependence agrees with the experimental cutoff lengths for strains from $0.3 \%$ to $10 \%$. Thus, any strain within this range will result in $\mathrm{Al}$ nanodots forming with size dependence similar to that shown in Fig. 3(b). Above $100 \mathrm{~nm}$, the island shapes change and do not follow the calculated dependence. Further, the top of the islands are parallel to the substrate and the sidewalls make angles ranging from $27^{\circ}$ to $35^{\circ}$ with the substrate plane. Wulff construction suggests that the island surfaces are composed of equilibrium (110) and (111) Al facets, consistent with the absence of a strong interface influence for films in the $>100 \mathrm{~nm}$ thickness regime. X-ray diffraction confirms predominantly (111) oriented Al.

We now turn our attention to the kinetic theory of roughening applied to scaling exponents observed in thin layers [Fig. 3(c)]. The growing surface is described in general by Eq. (3) with

$$
\begin{aligned}
F[h]= & \lambda_{1} \nabla^{4} h+\lambda_{2} \nabla^{2}(\nabla h)^{2}+\lambda_{3} \nabla^{2} h \\
& +\lambda_{4} \nabla^{2}\left[\sigma_{\perp}(h)^{2}-\sigma_{0}^{2}\right],
\end{aligned}
$$

where the last term addresses the effect of stress through the interface energy. ${ }^{20}$ Also accounted for in Eq. (4) are the $\nabla^{4} h$ Mullins term, to address dependence of the surface diffusion on local curvature, the CKPZ factor (not addressed in Ref. 20), and the Ehrlich-Schwoebel $\nabla^{2} h$ term. The importance of these effects will depend on the geometrical factor as well as the $\lambda_{i}$ coefficients. The Ehrlich-Schwoebel factor, for example, is weak in the case of Al (Ref. 21) and we may thus set $\lambda_{3}=0$. The remaining coefficients are $\lambda_{1}=-D \gamma$ and $\lambda_{4}$ $=2 D \sigma^{2} / E$, where $D=D_{s} \rho^{2} \tau / k_{B} T$, with $\rho$ the Al atomic volume and $\tau$ the atomic surface density, $D_{s}$ the surface diffusion coefficient, and $E$ the $\mathrm{Al}$ bulk modulus. The surface energy $\gamma$ is taken to be $\gamma_{t}$ and $\sigma$ is the average stress in the film. Values are included in Table I. For the stress obtained from our measurements, we find that $\lambda_{4} \ll \lambda_{1}$. We therefore do not expect the stress term in Eq. (6) to play a significant role in the scaling observed here. In the current context, the interface stress influences the formation of nanodots, as described above, and consequently contributes to the $\nabla^{4} h$ component. 
This analysis leaves us with the Mullins and CKPZ factors in Eq. (6). To obtain the scaling exponents we apply the standard transformations $x \rightarrow \mathrm{b} x, h \rightarrow b^{\alpha} h$, and $t \rightarrow b^{\beta / \alpha} t$ to Eq. (3), with the remaining terms of $F[h]$ from Eq. (6), and demand that $\partial h / \partial t$ be independent of $b$. For uncorrelated noise we use $\langle\eta(\mathbf{r}, t)\rangle=0$ and $\left\langle\eta\left(\mathbf{r}_{1}, t_{1}\right) \eta\left(\mathbf{r}_{2}, t_{2}\right)\right\rangle \propto \delta\left(\mathbf{r}_{1}\right.$ $\left.-\mathbf{r}_{2}\right) \delta\left(t_{1}-t_{2}\right)$. The incorporation of several terms in $F[h]$ leads to an overdetermined situation for $\alpha, \beta$, and ratio $\alpha / \beta$, which is generally resolved by examining the relative importance of each factor. When the Mullins term dominates over CKPZ, we obtain $\alpha=3 / 2$ and $\beta=3 / 8$. These are in good agreement with what we observe in the ultrathin regime, supporting the notion that the Mullins term dominates the surface properties for extremely thin $\mathrm{Al}$ films.

Even at modest temperatures surface self-diffusivity of $\mathrm{Al}$ is high. ${ }^{22}$ Diffusion of edge atoms at monolayer steps is accelerated by an exchange process. ${ }^{23}$ This argues that diffusional processes in room-temperature deposition of $\mathrm{Al}$ may not be ignored in examining the scaling properties, as noted in sputter deposition experiments. ${ }^{17}$ Although our AFM measurements do not address the surface properties at the atomic scale, the island formation reported here will be affected by the edge-growth process. This may explain our observation in Fig. 3(b) that the lateral correlation length grows faster than the average layer thickness for $\bar{h}<200 \mathrm{~nm}$. Furthermore, edge incorporation of $\mathrm{Al}$ atoms is consistent with selfaffine growth, as observed here. Lai and Das Sarma discuss situations in which $\alpha>1$ may occur. ${ }^{15}$ They suggest that this may be an unavoidable aspect of atomistic solid-on-solid growth at low temperatures, and relate the high roughness exponent to the formation of vacancies and overhangs. Stress due to the substrate is likely to play a role in these properties, although we expect it to be small.

It has not been established why dominance may switch from one term in the scaling functional to another during growth. In the present case, when island growth approaches equilibrium the highly sloped sidewalls appear to dominate the surface formation through the term $\lambda_{2} \nabla^{2}(\nabla h)^{2}$ in Eq. (6). From the scaling analysis of Eqs. (3) and (6) we obtain $\alpha$ $=1$ and $\beta=1 / 3$. These roughness and growth scaling exponents are in good agreement with what we observe for thicker Al layers. This supports our interpretation that the CKPZ mechanism is appropriate in describing the scaling of thick Al layers deposited at room temperature.

We examine the effect of stress for two cases. In the first case, coverage is uniform. Stress will be independent of thickness up to a critical relaxation thickness beyond which stress will relax via formation of dense dislocations. Below the relaxation thickness, coverage is smooth for this type of growth and the last term in Eq. (6) is negligible. Above the relaxation thickness, the effect of stress is trivial in the scaling properties. The second case is island formation, for which the stress within the islands is expected to show an exponential decay with distance from the film-substrate interface $\sigma_{0} \exp (-h / \ell)$. When $h$ is less than characteristic decay length $\ell$, the factor $\left[\sigma_{\perp}(h)^{2}-\sigma_{0}^{2}\right]$ in Eq. (6) approaches $(2 h / \ell) \sigma_{0}^{2}$. For situations in which this factor dominates over the other terms in $F[h]$, the scaling exponents are $\alpha=1 / 2$ and $\beta=1 / 4$. Such exponents may be relevant in cases where stress dominates, such as semiconductor nanodots formed heterogeneously on semiconductor substrates.

\section{SUMMARY}

Ultrathin Al layers are found to have a fine nanodot structure exhibiting lateral growth and increasing roughening with thickness. Both quantities saturate above $100 \mathrm{~nm}$ thickness. Additionally, the surface scaling exponent of the interface width rises rapidly and exceeds unity over a narrow thickness range, suggesting strong variations in the scaling properties not previously reported. The nanodot formation effects observed for thin layers are attributed to the presence of stress at the Al-substrate interface. Feature sizes are consistent with what is expected when the substrate induces nucleation of nanodots, although stress does not dominate the scaling exponent. Application of the kinetic theory of roughening adequately explains the high $(\alpha \sim 3 / 2)$ exponent when the $\nabla^{4} h$ Mullins term dominates. For thicker films the scaling exponent approaches $\alpha=1$, as expected for rough surfaces when ignoring interface effects. The surface equilibrium shapes obtained for the thick layers are consistent with faceted $\mathrm{Al}$ islands.

\section{ACKNOWLEDGMENTS}

The authors acknowledge support of this research by the National Science Foundation (CTS-0210141 and ECS0304224) and the J. F. Maddox Foundation.

${ }^{1}$ D. Aurongzeb, M. Basavaraj, K. Bhargava Ram, G. Kipshidze, B. Yavich, S. Nikishin, H. Temkin, and M. Holtz, J. Appl. Phys. 99, 014308 (2006).

${ }^{2}$ G. Palasantzas, T. Vystavel, S. A. Koch, and J. T. M. De Hosson, J. Appl. Phys. 99, 024307 (2006).

${ }^{3}$ M. Saitou, Phys. Rev. B 66, 073416 (2002).

${ }^{4}$ G. Palasantzas, S. A. Koch, and J. T. M. De Hosson, Appl. Phys. Lett. 81, 1089 (2002)

${ }^{5}$ V. A. Shchukin and D. Bimberg, Rev. Mod. Phys. 71, 1125 (1999).

${ }^{6}$ J. Krug, Adv. Phys. 46, 139 (1997).

${ }^{7}$ J. Tersoff and R. M. Tromp, Phys. Rev. Lett. 70, 2782 (1993).

${ }^{8}$ D. Aurongzeb, S. Pantibandla, M. Holtz, and H. Temkin, Appl. Phys. Lett. 86, 103107 (2005).

${ }^{9}$ R. Koch, D. Z. Hu, and A. K. Das, Phys. Rev. Lett. 94, 146101 (2005).

${ }^{10}$ E. Chason, B. W. Sheldon, L. B. Freund, J. A. Floro, and S. J. Hearne, Phys. Rev. Lett. 88, 156103 (2002).

${ }^{11}$ S. G. Mayr and K. Samwer, Phys. Rev. B 65, 115408 (2002).

${ }^{12}$ F. Family and T. Vicsek, J. Phys. A 18, L75 (1985).

${ }^{13}$ S. G. Mayr and K. Samwer, Phys. Rev. Lett. 87, 036105 (2001).

${ }^{14}$ C. V. Ramana, R. J. Smith, O. M. Hussain, and C. M. Julien, J. Vac. Sci. Technol. A 22, 2453 (2004).

${ }^{15}$ Z. W. Lai and S. Das Sarma, Phys. Rev. Lett. 66, 2348 (1991).

${ }^{16}$ A. I. Oliva, E. Anguiano, J. L. Sacedon, M. Aguilar, J. A. Mendez, and J. A. Aznarez, Phys. Rev. B 60, 2720 (1999).

${ }^{17}$ A. E. Lita and J. E. Sanchez, Phys. Rev. B 61, 7692 (2000).

${ }^{18}$ M. A. Lutz, R. M. Feenstra, P. M. Mooney, J. Tersoff, and J. O. Chu, Surf. Sci. 316, L1075 (1994).

${ }^{19}$ W.-C. Yang, H. Ade, and R. J. Nemanich, J. Appl. Phys. 95, 1572 (2004). ${ }^{20}$ Z. J. Liu and Y. G. Shen, Appl. Phys. Lett. 83, 5404 (2003).

${ }^{21}$ S. J. Liu, H. C. Huang, and C. H. Woo, Appl. Phys. Lett. 80, 3295 (2002).

${ }^{22}$ R. Stumpf and M. Scheffler, Phys. Rev. B 53, 4958 (1996).

${ }^{23}$ R. Stumpf and M. Scheffler, Phys. Rev. Lett. 73, 508 (1994).

${ }^{24}$ D. J. Siegel, L. G. Hector, and J. B. Adams, Phys. Rev. B 67, 092105 (2003).

${ }^{25}$ H. Mada, J. Chem. Phys. 75, 372 (1981).

${ }^{26}$ F. Y. Genin and W. J. Siekhaus, J. Appl. Phys. 79, 3560 (1996).

${ }^{27}$ CRC Handbook of Physics and Chemistry, 79th ed. (CRC, Boca Raton, FL, 1998). 